\title{
Estrogen and inflammation: hormone generous action spreads to the brain
}

\author{
Molecular Psychiatry (2002) 7, 236-238. DOI: 10.1038/ \\ $\mathrm{sj} / \mathrm{mp} / 4001007$
}

As direct targets of pharmaceuticals, estrogen receptors represent an attractive biological system for the discovery of potent, selective and specific drugs to control the evolution of selected disorders. ${ }^{1}$ In addition, their endogenous ligands, estrogens, were proved to faithfully fulfill the denomination of endocrine agents: the recent past featured a boost of novel physiological targets outside the hypothalamic-pituitary-ovary axis, the system where the action of sex hormones was believed to be restricted. To date, it appears that almost any tissue and cell in the human body is attracted by the fascinating power of estrogen, particularly the brain. A large body of studies points to a potential beneficial role of estrogens in different brain pathologies, as proved by epidemiological, experimental and animal model data after the natural or surgical ablation of estrogen synthesis. The role of estrogens in depression has been hypothesized for quite a long time based on the observation that decreased plasma levels of these molecules during the menstrual phase, after parturition or at menopause are often associated with mood disorders. Epidemiological studies have also suggested the involvement of hormonal factors in multiple sclerosis (MS): increased levels of sex hormones during pregnancy have been shown to associate with significant reduced severity of MS, whereas clinical symptoms often exacerbate postpartum, when sex hormone levels are remarkably reduced. Similarly, post-menopausal administration of estrogens may delay the onset of Alzheimer's disease (AD) in elderly women and ameliorate Parkinson's tremors. More recently, the comparison of the different age profile in the manifestation of psychotic episodes in the two sexes with the levels of circulating estradiol in normal and schizophrenic patients led to hypothesize a role for this hormone also in this CNS disorder.

Because of the diverse etiology of these diseases, the comprehension of the mechanism underlying estrogen beneficial action is puzzling. This issue is even more complex if we consider that hormone positively affects other pathophysiologic processes, such as osteoporosis, atherosclerosis and stroke.

Is there a common mechanistic denominator in all of these diseases which might underlie the widespread favorable effect of estrogens?

Correspondence: A Maggi, Via Balzaretti 9, 20133 Milan, Italy. E-mail: adriana.maggi@unimi.it
Inflammation has always been associated with the manifestation of MS and recent findings have underlined how the onset and progression of neurodegenerative diseases correlate with the existence of a local ongoing inflammatory reaction. Evidence has been provided that major depression associates with activation of the inflammatory response system and that antidepressants may reduce the interferon- $\gamma / \mathrm{IL}-10$ ratio, raising the question whether changes in the immune system and depression are causal or co-incidental effects. ${ }^{2}$ $\mathrm{AD}$ and cerebral ischemia are also associated with elevated expression of cytokines, adhesion molecules and other inflammatory molecules. ${ }^{3,4}$ Finally, increased levels of inflammatory cytokines were reported in psychiatric patients, revealing a potential role of these compounds also in schizophrenia. ${ }^{5}$ On the other hand, the role of inflammation in the pathogenesis of osteoporosis and atherosclerosis is well documented. As a consequence, the reduction of the inflammatory component is the aim of different therapeutic interventions or a beneficial complementary effect of drugs, used to cure these diseases.

Thus, an intriguing hypothesis to explain estrogen beneficial effects in so many and diversified human pathologies would be the existence of a link between hormone action and the machinery sustaining the inflammatory response.

Because of their well-known effect on ovarian follicle maturation, an inflammatory-like reaction, estrogens were considered more as pro- rather than antiinflammatory agents. However, besides the well established positive effects of sex hormones in selected pathologies with a relevant inflammatory component, such as MS and osteoporosis, there is now a wealth of experimental models where the lack of endogenous estrogens facilitates the onset of inflammation that is antagonized by estrogen replacement. Examples of such models are: carrageenan-induced pleurisy, endotoxin-induced uveitis, experimental encephalomyelitis and adjuvant-induced arthritis and cutaneous wound healing. ${ }^{6-8}$ In all of these models estradiol clearly opposes the inflammatory process.

Different biological mechanisms underlying estrogen anti-inflammatory activity have been proposed. Adhesion and migration of leukocytes through the endothelium of the vascular system are hampered by estradiol, as shown by the decrease in the expression of adhesion molecules (such as E-selectin, cadherins and CAM) and chemokines (such as the monocyte chemoattractant protein-1, MCP-1). A reduced synthesis in proteolytic enzymes might also account for estrogen reduction of tissue degradation and inflam- 
mation; proteases abundantly and specifically secreted by macrophages, such as cathepsins $\mathrm{K}$ and $\mathrm{L}$ involved in bone resorption, or matrix metalloproteinase-9 (MMP-9) implicated in matrix homeostasis and cell infiltration, are inhibited by estrogen receptor activation. Extensive work on estrogen action in bone remodeling demonstrated that the estrogen protective role in inflammatory processes also occurs by blocking resident inflammatory cell production of proinflammatory cytokines (IL-1, IL-6 and TNF- $\alpha$ ), hematopoietic growth factors (macrophage colony stimulating factor, M-CSF) and cell differentiation agents (receptor activator of NF-kB ligand, RANKL). Therefore, inhibition of the synthesis of these potent inflammatory mediators has been proposed to explain the detrimental effects of estrogen deficiency on the human skeletal system at menopause. Consistently with this hypothesis, estradiol has been shown to oppose the inflammatory reaction occurring in carrageenan-induced pleurisy by blocking the synthesis of proinflammatory mediators, such as IL-6, TNF- $\alpha$ and iNOS. The inhibitory regulation of estradiol on the synthesis of iNOS induced by inflammatory stimuli has been observed in several cellular and biological systems, suggesting a primary role for this pathway in estrogen biological activity.

Since macrophages play an essential role in the inflammatory process, the positive effect of estrogen on inflammation-related diseases could be mediated by suppressing monocyte recruitment and/or macrophage activation at the inflammatory site.

Microglia, the resident immune cells in the brain, play a pivotal role in immune surveillance, host defense and tissue repair in the CNS. In response to brain injury or immunological challenges, microglia readily become activated as characterized by morphological changes, expression of surface antigens and production of immune modulators. ${ }^{9}$ The precise function of activated microglia and their secretory products remains controversial. The initial phase of microglia response aimed at inducing phagocytosis has relevance in sustaining neuron viability, removing toxic cellular debris and promoting tissue repair and is a critical event in the resolution of the inflammatory attack. On the other hand, a persistent state of microglia activation has been hypothesized to have negative effects of neuron viability. Microglia activation is in fact a histopathological hallmark of several neurodegenerative diseases, including Parkinson's disease, AD, MS, AIDS dementia, Huntington, Sandhoff disease and Lewy bodies dementia, suggesting its cytotoxic potential. These observations led us to hypothesize that a chronic inflammatory reaction, driven mainly by reactive microglia, may contribute to the process of neuronal loss and matrix destruction observed in these chronic disorders.

Recently, two groups have independently shown that estradiol has a strong influence on microglia activity. ${ }^{10,11}$ Estrogen in fact opposes the morphological and biochemical activation of microglia induced by the bacterial endotoxin lipopolysaccharide (LPS). This effect, observed in primary cultures of rat microglia with very low concentration of $17 \beta$-estradiol, is dosedependent and blocked by specific ER antagonists. These observations, together with the finding that cells must be challenged with hormone for a few hours in order to become irresponsive to LPS, suggest that a receptor-mediated mechanism is involved. Indeed, rtPCR and immunocytochemical studies reported in these recent papers show the presence of high concentrations of $\mathrm{ER} \alpha$ and $\mathrm{ER} \beta$ in microglia, in agreement with previous reports on receptor expression in monoblastoid and macrophage cells. ${ }^{12}$

Existing evidence on estrogen activity in 'in vivo' models of CNS inflammatory disorders are still scarce, albeit the investigations made so far on experimental autoimmune encephalomyelitis (EAE) and ischemic damage are in line with the hypothesis of an antiinflammatory role of estrogens. EAE is an inflammatory demyelinating disease of the CNS induced by immunizing susceptible strains of laboratory rodents with myelin proteins or peptides and is a model that considerably contributed to gain insights into the pathogenesis of MS. In this model, gender difference in the susceptibility and severity parallel gender differences reported in MS and treatment with low doses of $17 \beta$ estradiol (comparable with diestrus levels) significantly reduced the severity of EAE by decreasing the number and size of inflammatory and demyelinated foci and the secretion of pro-inflammatory cytokines, while augmenting the secretion of anti-inflammatory cytokines (IL-10); ovariectomy, conversely, caused significantly more severe EAE. Estrogens were also shown to be protective in case of ischemic damage in different models of CNS injury. It has been proposed that estrogens exert part of their protective effects by preventing reperfusion associated with permanent brain damage, which produces an influx of oxygen followed by an accumulation of oxygen-derived free radicals; estradiol attenuates the peroxidative damage and iNOS induction.

In the last few years several experimental reports have shown that estrogens protect against brain degeneration by directly targeting neurons. ${ }^{13}$ The recent findings on estrogen-dependent attenuation of microglia response provide a novel explanation for hormone action in the brain and for the understanding of the neurodegenerative process in neural pathologies, like MS, AD or ischemic brain damage, in which inflammation plays a relevant role, and eventually also in schizophrenia or depression, where suggestive evidence indicates their association with local inflammation.

An interesting issue of both biological and pharmaceutical relevance is the unraveling of the molecular players influenced by estradiol in the inflammatory plot. The primary targets of estradiol action within responsive cells are the two well-described receptors (ER- $\alpha$ and ER- $\beta$ ) which are hormone-regulated transcription factors. Hormone-activated receptors can induce or repress transcription, in a cell and promoter context-dependent manner, through the interaction with multisubunit complexes containing coactivator 
and/or corepressor functions. Many observations have indicated that estradiol directly inhibits the expression of pro-inflammatory genes via a receptor-mediated mechanism, in spite of the lack of canonical estrogen responsive elements in the promoter region of these genes. Because of the capability of hormone-activated ERs to interact with other classes of transcription factors coupled to membrane receptor signaling molecules (such as Sp-1, Jun/Fos, NF-kB and Stat), it is postulated that estrogen-dependent inhibition of transcription occurs without a physical interaction of the ligandreceptor complex with the proinflammatory gene promoters. The receptor could in fact alter the dynamics of the association of other transcription factors with the transcription machinery (a mechanism known as transrepression). This would be reminiscent of the mechanism evoked for the glucocorticoid receptor (GR) and the peroxisome proliferator activated receptors (PPAR) $\alpha$ and $\gamma$, which also have been shown to inhibit macrophage activation and production of pro-inflammatory cytokines at the level of transcription.

Alternatively, transrepression could be the result of the competition between NRs and other classes of transcription factors for limiting amounts of general coactivators, such as CREB-binding protein (CBP) and p300, or for NR specific activators such as steroids receptor coactivator-1 (SRC-1). Recent findings have suggested that recruitment of complexes bearing histone deacetylase activity provides a critical step in the process of transrepression by NRs; thus, inhibition of histone acetylation could represent an additional level of control of pro-inflammatory gene expression. ${ }^{14}$ Future research will be devoted toward the understanding of whether and which of these mechanisms hold true for the ER anti-inflammatory activity.

ER knock-out animal models have demonstrated that, at least in the brain and vascular wall, the selective activation of the $\alpha$-isoform is responsible for mediating the protective effects of estrogen. However, nongenomic actions accounting for rapid effects both in the onset and duration have been described and associated with an estrogen receptor lining in the plasma membrane; limited existing information on this receptor structure and on the relative signal transduction pathway have however hampered the understanding of the biological and pharmacological implications.

It is too early to think of estrogen as a truly antiinflammatory pill; however, it is tempting to speculate on its potency and mechanism of action. Would estrogen stand the comparison with cortisone, the prototypic anti-inflammatory compound acting through NRs? A comparison between the activity of these two classes of drugs would have relevant biomedical consequences, in both the acute treatment of infections (feminine secondary effects are null here) and in chronic preventive therapies (for which active industrial effort is elaborating ER drugs at their third generation as tissue-specific selective estrogen receptor modulators, or SERMs). A SERM is a molecule that binds with high affinity to the ER and has tissue-specific effects distinct from estradiol, acting as an estrogen agonist in some tissues and as an antagonist in others. The development of SERMs that selectively interact with specific receptors in specific tissues offers the possibility of improving the risk/benefit profile of hormone replacement therapy and perhaps even extending the use of these analogues to men, for whom the usefulness of estrogen therapy has been limited by its potential feminizing effects. Academic, industrial and public attention is devoted towards the future expansion and innovations in the selective modulation of ER to prevent chronic diseases and promote healthy aging.

At menopause, the ovarian synthesis of estrogen is over and women face the possibility of living the last third of their life with mild or severe menopausal syndrome. At best, some will experience mucosal (vaginal) atrophy and vasomotor disturbance; others do carry the risk of developing a chronic disabling or disqualifying disease, making the choice of hormone replacement therapy more critical. By extending also to the brain, the prodigious activity of estrogen has brought high expectations as to the beneficial role of hormone in the preventive therapy and health of the aging women.

E Vegeto, P Ciana, A Maggi Center of Excellence on Neurodegenerative Diseases University of Milan 20133 Milan, Italy

1 Emery GJ et al. Trends Pharmacol Sci 2001; 22: 233-240.

2 Licinio J, Wong ML. Mol Psychiatry 1999; 4: 317-327.

3 Gonzalez-Scarano F, Baltuch G. Ann Rev Neurosci 1999; 22: 219-240.

4 Iadecola C, Alexander M. Curr Opin Neurol 2001; 14: 89-94.

5 Muller N et al. Ann NY Acad Sci 2000; 917: 456-467.

6 Cuzzocrea S et al. Endocrinology 2000; 141: 1455-1463.

7 Miyamoto N et al. J Immunol 1999; 163: 374-379.

8 Jansson L et al. J Neuroimmunol 1994; 53: 203-207.

9 Kreutzberg GW. Trends Pharmacol Sci 1996; 8: 312-318. 10 Bruce-Keller AJ et al. Endocrinology 2000; 141: 3646-3656. 11 Vegeto E et al. J Neurosci 2001; 21: 1908-1918.

12 Vegeto E et al. FASEB J 1999; 13: 793-803.

13 Toran Allerand CD et al. Front Neuroendocrinol 1999; 20: 97-121. 14 Glass CK, Rosenfeld MG. Genes Develop 2000; 14: 121-141. 